\title{
Metodología enfocada en competencias
}

Saúl Campos Morán Investigador UTEC

\section{RESUMEN}

Este artículo trata sobre la educación basada en competencias y sustenta la propuesta de un enfoque metodológico que promueva su implementación en el proceso educativo. Existen diferentes corrientes teóricas que plantean varias clasificaciones de las competencias, las cuales, a nivel general, distinguen dos tipos de éstas, las genéricas y las específicas, las cuales a su vez tienen más subdivisiones especializadas, pero hace falta un modelo que las integre como eje transversal en la educación. La metodología apoyada en este artículo plantea la posibilidad que estos diferentes tipos de competencia formen parte del proceso educativo.

\section{$\mathbf{L}$} a educación contemporánea se complementa con varias disciplinas, creando la necesidad en los profesionales salvadoreños y de todo el mundo de tener los conocimientos y actitudes para afrontar el reto de enseñar adecuadamente. Esto implica cambiar supuestos básicos de la docencia convencional y aplicar conocimientos y enfoques educativos que permitan un desarrollo integral de los individuos no solo como profesionales, sino también educación para el mundo en el cual se desenvuelven. Varios proyectos como Tuning (2007), ISFOL (2003), DESECO (2004), entre otros, han propuesto enfoques educativos universitarios basados en competencias que proporcionan un marco referencial de educación aplicada al desarrollo de las capacidades de los alumnos, permitiendo clasificar y dar seguimiento al desarrollo de dichos conocimientos y actitudes. En El Salvador, el modelo basado en competencias es aplicado, pero, ¿Qué son las competencias?, ¿hasta que punto los egresados de las universidades adquieren y desarrollan las competencias propuestas por dichos proyectos? ¿Podemos hablar de una educación basada en competencias en El Salvador?

Según Espinoza y Reves (2003), la educación no debe ser enfocada solo como una institución al servicio del aparato productivo por cuanto sus funciones rebasan con creces una mera funcionalidad económica, cumpliendo un papel estratégico en el logro de la identidad nacional, la integración social, la realización cultural, individual y colectiva, así como el desarrollo de la personalidad y de actitudes y comportamientos de convivencia social. En el mismo texto, Figueroa (2000), citado por Espinoza y Reyes (2003), sostiene que el objetivo fundamental del proceso educativo es el fortalecimiento de la personalidad a través de la formación y desarrollo de los aspectos moral, intelectual y físico, entre otros, con el propósito de formar 


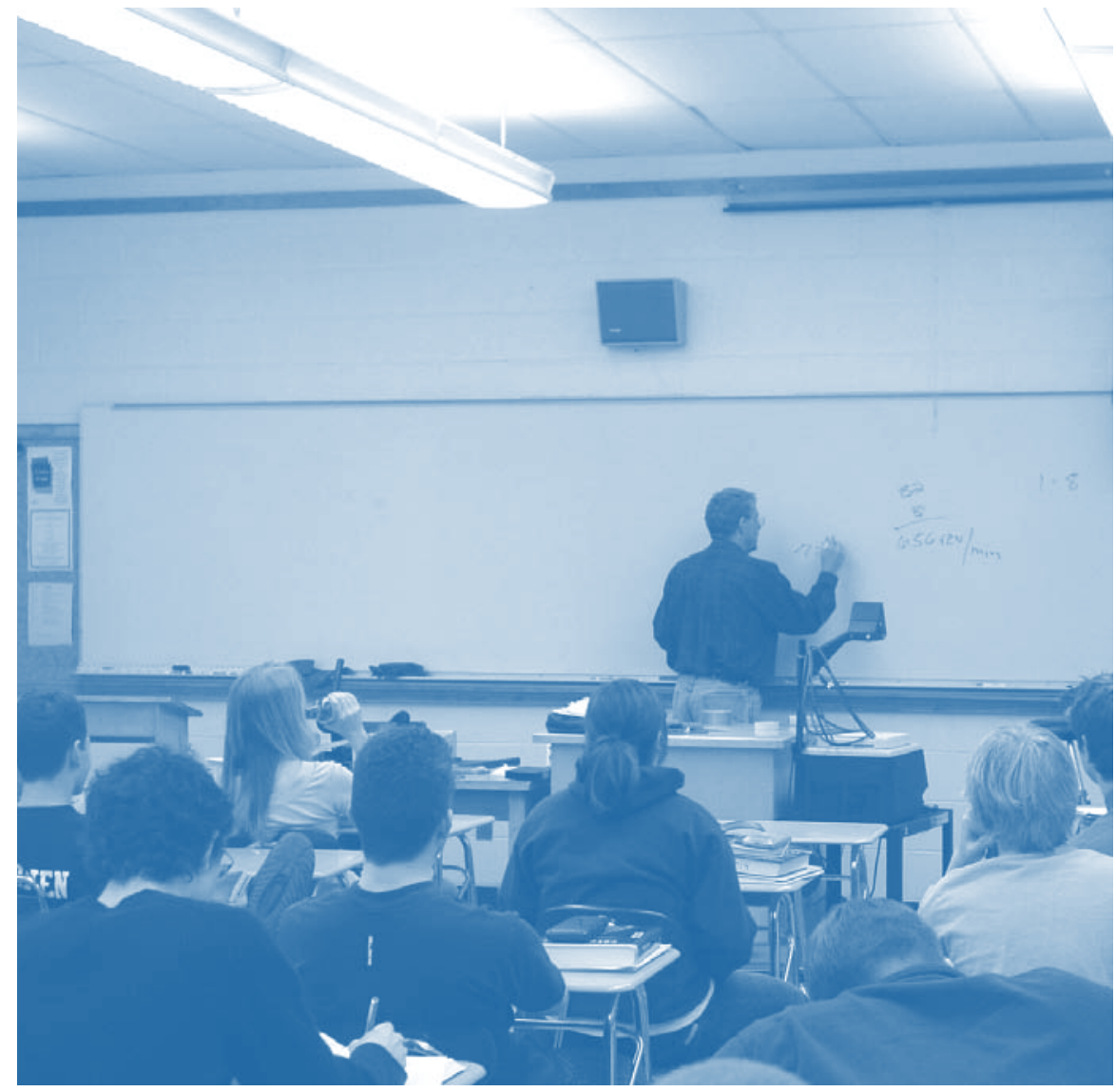

personas de alta calidad humana, capaces de elevar la calidad de vida del planeta en su conjunto.

Actualmente las competencias académicas juegan un papel preponderante en el ámbito educativo. De acuerdo con Larrain (2005), hablar de "Educación basada en competencias" implica cambios radicales en el que hacer educativo, cambios desde la orientación social de la práctica educativa.

Términos como capacidad, atributo, habilidad, destreza y competencia tienden a usarse indistintamente entre ellos, ya que su significado tiene un cierto grado
En este contexto, la competencia es la herramienta que le permite al individuo desempeñarse integralmente en sus actividades tanto a nivel general como de su rubro específico. Se refiere a que una persona pone en práctica determinada capacidad o habilidad para desempeñar una labor y que puede hacerlo de un modo que permita evaluar el nivel de consecución (Escudero Muñoz, 2007). Esta evaluación es posible porque las personas no poseen una competencia en términos absolutos, sino en grados, de ahí que éstas se pueden desarrollar mediante el ejercicio y la educación.

Diversas investigaciones han demostrado, como revisan Gaviria y Fernández (2006), citados por Lucas Mangas (2004), que las metas orientadas hacia el desarrollo de competencias (es decir, hacia el dominio de una determinada área de actividad o conocimiento) valoran más la mejora personal que se puede lograr con la actividad que los resultados evaluables por criterios externos. De ahí su mayor relación con una motivación intrínseca (por la acción misma), que con una motivación extrínseca (medio para conseguir otra meta).

(2005), por competencias se entiende la concatenación de saberes, no solo pragmáticos y orientados a la producción, sino aquellos que articulan una concepción del ser, del saber, saber hacer y saber convivir.
Dentro de un sistema educativo tradicional, los programas están centrados las materias correspondientes a los dominios básicos del conocimiento. Es deen temas que constituyen cada una de 
cir, los programas indican "sobre qué" han de aprender los alumnos. El criterio de logro se centra en los contenidos temáticos de las materias. En este mismo modelo, el aprendizaje de los contenidos de las materias generalmente se reduce a la reproducción literal de la información. La interacción que los alumnos establecen con los contenidos programáticos generalmente es con las características morfológicas del lenguaje, oral o escrito, mediante el cual se les "transmite" la información.

En la otra mano, la competencia es un saber hacer con conciencia. Es un saber en acción, un saber cuyo sentido inmediato no es "describir" la realidad, sino "modificarla", no definir problemas sino solucionarlos; un saber el qué, pero también un saber cómo (Larrain, 2005). Las competencias son, por tanto, propiedades de las personas en permanente modificación que deben resolver problemas concretos en situaciones de trabajo con importantes márgenes de incertidumbre y complejidad técnica.

Las competencias son estructuralmente capaces de transferir su valor a diferentes campos, así como generar conocimiento y nuevas competencias dinámicamente. Estas tienen múltiples facetas, como la epistemológica, sociológica, política y práctica (Escudero Muñoz, 2007); y por ello reclaman una perspectiva multidimensional e inte- gral de los aprendizajes. Comprender y movilizar diversos recursos cognitivos, personales, sociales y éticos constituye la estructura de una competencia, así como la capacidad de aprender a aprender, que es esencial para captar su carácter cambiante y evolutivo.

En educación, el concepto de competencia se presenta como una red conceptual amplia, que hace referencia a una formación integral del ciudadano por medio de nuevos enfoques, como el aprendizaje significativo, en diversas áreas (Larrain, 2005): cognoscitiva, (saber), psicomotora (saber hacer, aptitudes), afectiva (saber ser, actitudes y valores). En este sentido, la competencia no se puede reducir al simple desempeño laboral, tampoco a la sola apropiación de conocimientos para saber hacer, sino que abarca todo un conjunto de capacidades, que se desarrollan a través de procesos que conducen a la persona responsable a ser competente para realizar múltiples acciones (sociales, cognitivas, culturales afectivas, laborales, productivas), por las cuales proyecta y evidencia su capacidad de resolver un problema dado, dentro de un concepto específico y cambiante. Así, la formación integral se va desarrollando poco a poco, por niveles de complejidad, en los diferentes tipos de competencias básicas: básicas o fundamentales, genéricas o comunes, específicas o especializadas y laborales. La competencia, al igual que la inteligencia, no es una capacidad innata, sino que, por el contrario, es susceptible de ser desarrollada y construida a partir de las motivaciones internas de cada cual, motivaciones que deberán ser comunicadas al grupo de trabajo.

Las competencias se tienen que aprender en la acción. Según Evequoz (2003), citado en el estudio del grupo Bitácola en 2006, se adquieren en la diversidad de momentos que van más allá del marco de la profesión y se extienden en la vida asociativa, la esfera familiar, la práctica del deporte o de una actividad cultural. Por lo tanto la universidad puede promover situaciones de aprendizaje y proporcionar guías que clarifiquen dónde y cómo el estudiante puede incrementar o mejorar sus competencias genéricas.

\section{Una metodología basada en competencias}

Para poder hablar de una metodología basada en competencias es necesario, primero, contar con docentes que dispongan de dichas competencias para ser capaces de evocar las mismas en los alumnos. De tal forma, la metodología misma debe ser considerada como competencia (UPM 2005), tomando en cuenta componentes de orden sistémico e instrumental como los mencionados anteriormente. 
Bennet, Dunne y Carré (1999) disciernen, según contenidos, si el aprendizaje de las competencias genéricas tiene que asociarse más al entorno académico o a las experiencias de trabajo simuladas o directas, o vehicularlas a través de proyectos. En su artículo citan a Dummond y otros (1997) que consideran tres enfoques de tratamiento de las competencias genéricas en la enseñanza superior: infundido o integrado en el currículum, cursos paralelos o independientes y conexión con el puesto de trabajo o proyectos basados en el trabajo.

Entonces, de acuerdo a estas tendencias, vamos a retomar el enfoque por competencias propuesto por Cepeda (2004), el cual plantea que la metodolo- gía para potenciar el enfoque por competencias en la educación debe distinguir los siguientes pasos:

1. Evaluación de necesidades, en la que se van a fijar los supuestos teóricos-previos en los que se basará dicha evaluación (destinatarios, receptores de la evaluación, finalidad, propósitos, objetivos de la evaluación, papel del evaluador, etc.)

En aspectos, junto con las características del programa y las del contexto en que se va a llevar a cabo el mismo, nos servirán para determinar el modelo y tipo de evaluación en el diseño de ésta, una vez que se valide el programa y podamos pasar a precisar la metodología de evaluación.
- Justificación.

- Competencias necesarias.

- Qué tipo de competencias es prioritario a desarrollar en el alumno, en esta materia.

- Cómo aplicar dichas competencias en una situación real de trabajo.

- Utilidad que representan dichas competencias en el desarrollo profesional y social.

- Importancia de obtener ciertas habilidades o competencias adicionales.

2. Especificación de competencias, en la cual se pretenden establecer las características de la calidad formal/intrínseca del programa, la adecuación/adaptación al contexto y la aceptación en el mismo.

Para este análisis se necesita tener en cuenta, por una parte, la relación entre los rasgos del profesional que se pretende formar, y las necesidades sociales dadas y, por otra, los cambios que haya habido en conocimientos científicos y en las tecnologías que el egresado se supone dominará y utilizará en el ejercicio de su profesión (ISFOL 2003).

Los enunciados de competencias deben ser:

- Específicos

- Reales

- Que precisen una habilidad 
- Redactados en tiempo presente

- Jerarquizados por áreas de conocimiento:

- Definir qué es lo que el educando necesita de manejar de esa rama del conocimiento.

- Definir cuánto se la va a dar de cada área dependiendo de las características de los educandos, la carrera y necesidades de egreso.

\section{Determinación de componentes;} en esta tercera fase se persigue específicamente responder a las competencias principales para el desempeño profesional y establecer los criterios para que el programa reúna las condiciones para poder ser evaluado. Es decir, en este apartado vamos a elegir el modelo a implementar en cuanto a la concepción de competencias. En este sentido, nuestra propuesta nos lleva a retomar la clasificación propuesta por Tuning (2007), que dicta que las competencias pueden ser:

Instrumentales: Aquí se incluyen todas aquellas competencias que ofrecen a los alumnos los conocimientos y habilidades para enfrentarse a sus problemas técnicos del día a día. A su vez, estas se dividen en:

- Habilidades cognoscitivas, la capacidad de comprender y manipular ideas y pensamientos.
- Capacidades metodológicas para manipular el ambiente: ser capaz de organizar el tiempo y las estrategias para el aprendizaje, tomar decisiones o resolver problemas.

- Destrezas tecnológicas relacionadas con el uso de maquinaria, destrezas de computación y gerencia de la información.

- Destrezas lingüísticas tales como la comunicación oral y escrita o conocimiento de una segunda lengua.

Interpersonales: Capacidades individuales de expresar los sentimien- tos, y habilidades críticas y de autocrítica. Son destrezas sociales como la capacidad de trabajar en equipo o la expresión de compromiso social. Facilitan los procesos de interacción social.

Sistémicas: Son las destrezas y habilidades que conciernen a los sistemas como totalidad. Suponen una combinación de la comprensión, la sensibilidad y el conocimiento que permiten al individuo ver como las partes de un todo se relacionan y se agrupan. Estas capacidades incluyen la habilidad de planificar los

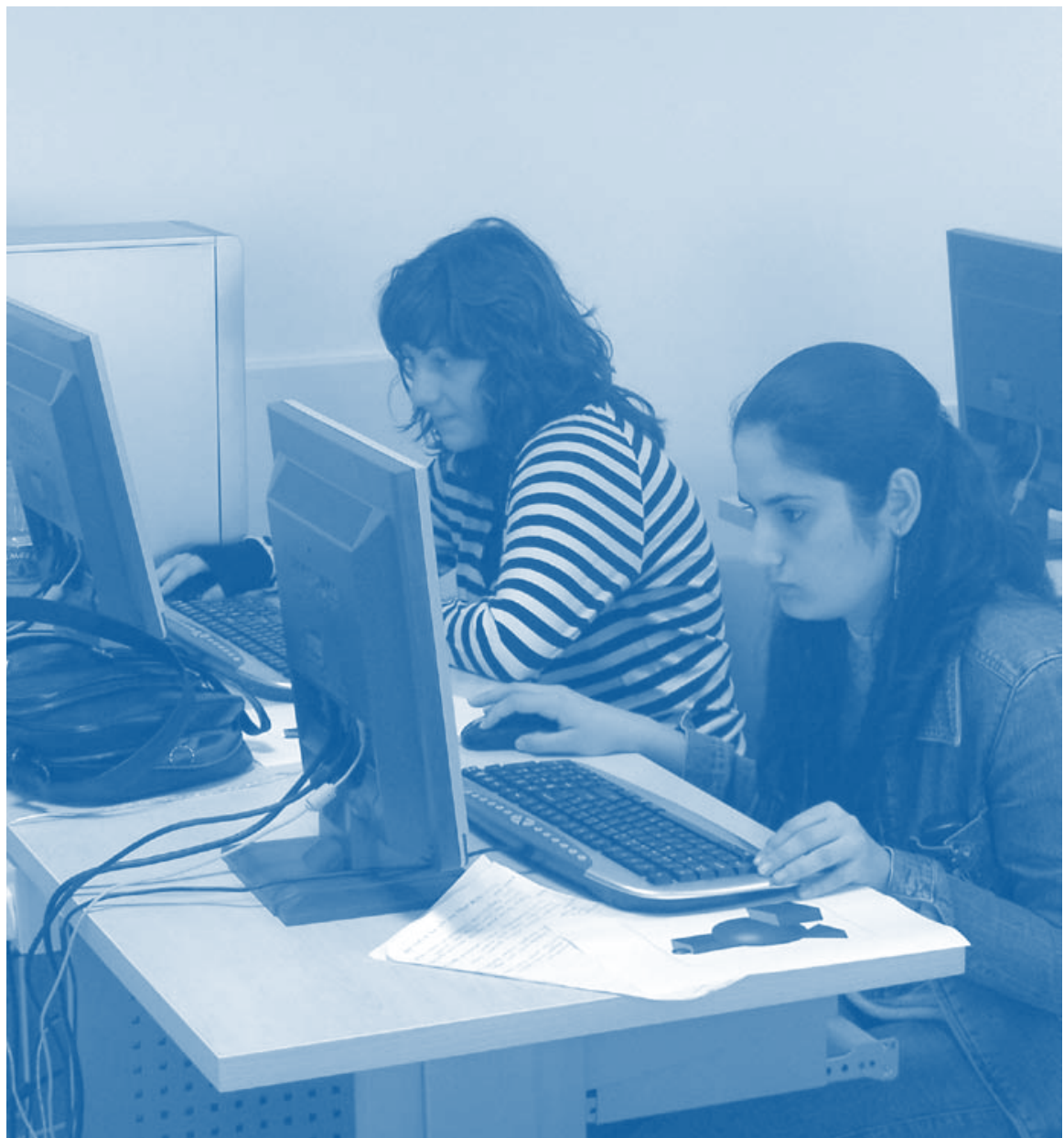


cambios de manera que puedan hacerse mejoras en los sistemas como un todo y diseñar nuevos sistemas. Las competencias sistémicas o integradoras requieren una combinación de comprensión, sensibilidad y conocimientos; para ello es preciso adquirir previamente competencias instrumentales e interpersonales.

\section{Identificación de procedimientos} para el desarrollo de competencias, se pretende establecer los recursos didácticos, métodos de enseñanza, experiencias vivenciales y la utilización de audiovisuales, y otros aspectos que buscan la identificación de las actividades diseñadas, y que nos permiten establecer los indicadores de evaluación. Éstas, según Cepeda, pueden ser:

\section{- Cobertura del programa.}

- Realización de actividades: Se han llevado a la práctica todas y cada una de las actividades planeadas.

- Ejecución de la temporalización: Muy relacionada con la anterior, hace referencia que se han llevado a cabo las actividades previstas en los períodos de tiempo prefijado, es decir hay un ajuste entre la ejecución real y la planeación diseñada.

- Funciones de los agentes implicados: Cada agente implicado en el programa ha llevado a la práctica

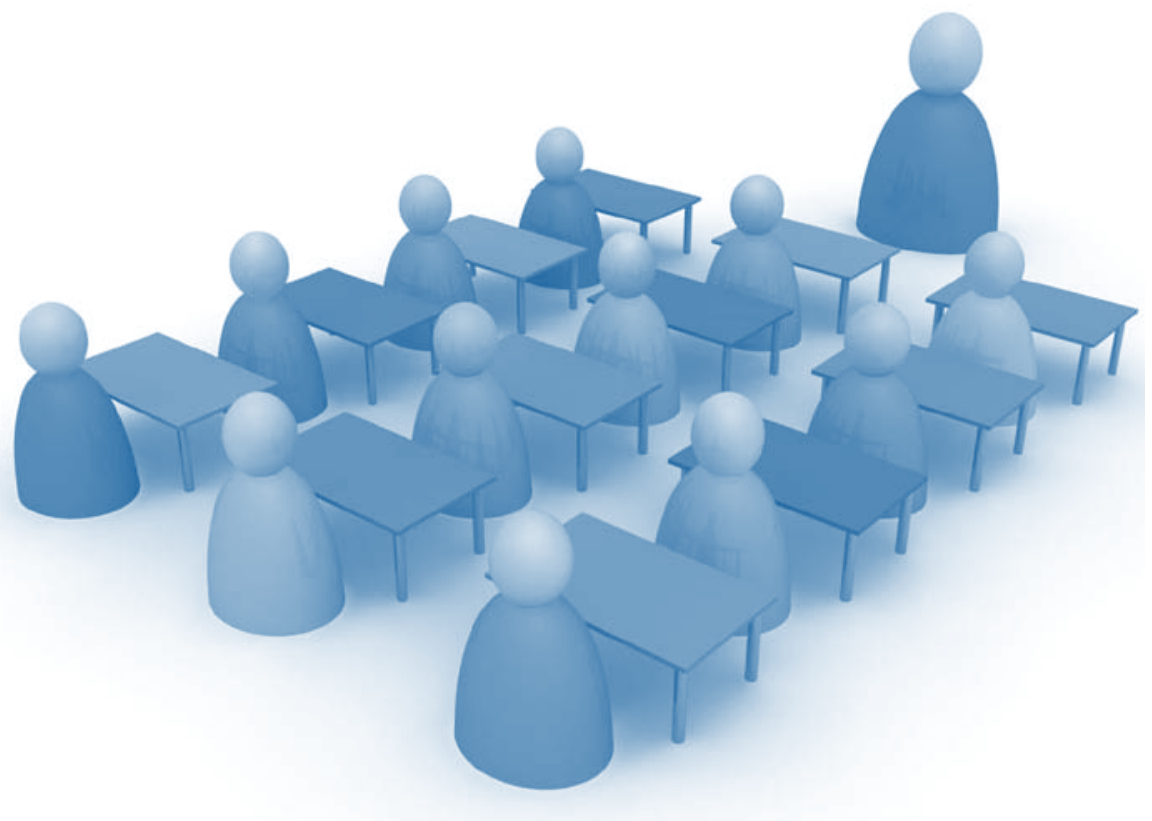

las actividades de las que era responsable, tal y como se diseñó.

- Utilización de los recursos disponibles: Se han empleado los recursos materiales y humanos que se habían previsto.

5. Definición de evaluación de competencias. Igual que la metodología, este elemento estructural de los programas está dispuesto para el docente a nivel de políticas y algunas sugerencias.

Los elementos de un programa de contenidos tomados en cuenta hasta este momento, son esenciales para la dosificación.

\section{Validación de competencias pro-} cedimientos y medios de evaluación, la última fase de este modelo, en que se trata de realizar una evaluación de resultados del logro de Ios niveles de aprendizaje y efectos del programa.

\section{Conclusión}

El enfoque por competencias requiere de docentes que cuenten con la capacidad de crear y adaptar metodologías que involucren al estudiante como protagonista del desarrollo de sus propias potencialidades sociales, espirituales y físicas; para ello es necesario un dominio de por lo menos una de las diferentes propuestas teóricas sobre dicho 
enfoque para luego poder plasmar en el modelo a implementar dicho referente, el cual debe estar acorde a la realidad política, social y económica en la que vivimos, tropicalizando y adaptándolo al entorno.

Para que las competencias se desarroIlen es necesario contar con un modelo que identifique cuales son las habilidades, conocimientos y actitudes que realmente son necesarios en la sociedad, luego es importante determinar como vamos a medirlo, qué actividades haremos para potenciarlas y por supuesto, contar con un instrumento de evaluación que nos permita un seguimiento transversal, ya que evaluar por competencias implica evaluar integralmente a un individuo, puesto que aprenderlas implica un aprendizaje para la vida.

\section{BIBLIOGRAFÍA}

- BENETT, N., DUNNE, E. \& CARRE, C. (1999). Patterns of core and generic skill provision in higher education. Higher Education, 37(1), 71-93.

Disponible en:

http://books.google.es/books?id=VE k9AAAAIAAJ\&pg=PA99\&dq=higher + education+bennett+dunne+carre\&c $\mathrm{d}=10 \# \mathrm{v}=$ onepage $\& \mathrm{q}=$ higher\%20edu cation\%20bennett\%20dunne\%20car re\&f=false consultado el 15/04/10
- CEPEDA, Martín. Metodología de la enseñanza basada en competencias. Revista Iberoamericana de educación, ISSN: 1681-5653, disponible en http://www.rieoei.org/ deloslectores/709Cepeda.PDF, consultado en $15 / 04 / 10$

ESCUDERO MUÑOZ, Juan M. LaS competencias profesionales y la formación universitaria: posibilidades y riesgos. Red U. Revista de Docencia Universitaria, número 1 http://www. redu.um.es/Red_U/1/ Universidad de Murcia. jumaes@um.es Consultado el 04/02/2009

- ISFOL, II punto su... certificazione delle competenze (The point about... competence certification), 2003 Disponible en www.isfol.it/DocEditor/ test/File/Studi_Isfol_Spa_5.pdf, consultado en $04 / 13 / 10$

- Lucas Mangas, Susana. Desarrollo de las competencias: "Preocupación por la calidad" y "motivación de logro" desde la docencia universitaria. Red U. Revista de Docencia Universitaria. Num. 2 http://www.um.es/ ead/Red_U/2/, Universidad de Valladolid, consultado el 06/16/09

MONTERO Lagos, Patricio, PhD. Desafíos para la profesionalización del nuevo rol docente universitario, Universidad de Santiago de Chile
Norelkys Espinoza y Maricarmen Pérez Reyes. La Formación Integral del Docente Universitario. FERMENTUM Mérida - Venezuela - ISSN 0798-3069 - AÑO 13 - No 38 - SEPTIEMBRE - DICIEMBRE - 2003 - 483-506

- Proyecto Tuning América Latina, Informe final. Reflexiones y perspectivas de la Educación Superior en América Latina, editado por Beneitone, Pablo, Esquetini, César, González, Julia, Marty Maletá, Maida, Siufi, Gabriela, Wagenaar, Robert, 20042007

- Taller de Estudio de Competencias Transversales para el Grado en Ingeniería Informática, Escuela Superior de Ingeniería Informática, Universidad de Sevilla, Junio 2008

UPM. Nuevas metodologías Docentes. Disponible en http://www.upm. es/innovacion/cd/02_formacion/taIleres/nuevas_meto_docent/nuevas_metodologias_docentes.pdf, consultado en 15/04/10

WAGENAAR, Robert. Una introducción a Tuning Educational Structures in Europe La contribución de las universidades al proceso de Bolonia, Brochure general del Proyecto Tuning, Proyecto Tuning, 2007. Disponible en http://tuning.unideusto.org/ tuningeu, consultado el 03/18/2009 